\title{
SOME GRAPHS DETERMINED BY THEIR SIGNLESS LAPLACIAN (DISTANCE) SPECTRA*
}

\author{
CHANDRASHEKAR ADIGA ${ }^{\dagger}$, KINKAR CHANDRA DAS ${ }^{\ddagger}$, AND RAKSHITH B. R. ${ }^{\S}$
}

\begin{abstract}
In literature, there are some results known about spectral determination of graphs with many edges. In [M. Cámara and W.H. Haemers. Spectral characterizations of almost complete graphs. Discrete Appl. Math., 176:19-23, 2014.], Cámara and Haemers studied complete graph with some edges deleted for spectral determination. In fact, they found that if the deleted edges form a matching, a complete graph $K_{m}$ provided $m \leq n-2$, or a complete bipartite graph, then it is determined by its adjacency spectrum. In this paper, the graph $K_{n} \backslash K_{l, m}(n>l+m)$ which is obtained from the complete graph $K_{n}$ by removing all the edges of a complete bipartite subgraph $K_{l, m}$ is studied. It is shown that the graph $K_{n} \backslash K_{1, m}$ with $m \geq 4$ is determined by its signless Laplacian spectrum, and it is proved that the graph $K_{n} \backslash K_{l, m}$ is determined by its distance spectrum. The signless Laplacian spectral determination of the multicone graph $K_{n-2 \alpha} \vee \alpha K_{2}$ was studied by Bu and Zhou in [C. Bu and J. Zhou. Signless Laplacian spectral characterization of the cones over some regular graphs. Linear Algebra Appl., 436:3634-3641, 2012.] and Xu and He in [L. Xu and C. He. On the signless Laplacian spectral determination of the join of regular graphs. Discrete Math. Algorithm. Appl., 6:1450050, 2014.] only for $n-2 \alpha=1$ or 2. Here, this problem is completely solved for all positive integer $n-2 \alpha$. The proposed approach is entirely different from those given by Bu and Zhou, and $\mathrm{Xu}$ and $\mathrm{He}$.
\end{abstract}

Key words. Signless Laplacian spectrum, Distance spectrum.

AMS subject classifications. $05 \mathrm{C} 50$.

1. Introduction. Graphs considered in this paper are all simple. Let $G$ be a graph with vertex set $V(G)=\left\{v_{1}, v_{2}, \ldots, v_{n}\right\}$ and edge set $E(G)$. The degree of a vertex $v_{i}$ is the number of neighbors of $v_{i}$ in $G$ and is denoted by $d_{i}(G)$. Throughout the paper, we assume that the sequence $\left\{d_{i}(G)\right\}_{i=1}^{n}$ is non-increasing, i.e., $d_{i}(G) \geq d_{i+1}(G), i=1,2, \ldots, n-1$. The adjacency matrix of $G$, denoted by $A(G)$, is the $n \times n$ real symmetric matrix whose $(i, j)$-entry is 1 if $v_{i} v_{j} \in E(G)$ and 0 otherwise. The adjacency spectrum or spectrum of $G$ is the multiset of eigenvalues of $A(G)$. The matrix $L(G)=D_{g}(G)-$ $A(G)$ (resp., $\left.Q(G)=D_{g}(G)+A(G)\right)$, where $D_{g}(G)=\operatorname{diag}\left(d_{1}(G), d_{2}(G), \ldots, d_{n}(G)\right)$ is the Laplacian matrix (resp., signless Laplacian) of $G$ and the $L$-spectrum (resp., Q-spectrum) of $G$ is the spectrum of $L(G)$ (resp., $Q(G)$ ). Two graphs are cospectral (resp., $L$-cospectral, $Q$-cospectral) if they have same spectrum (resp., $L$-spectrum, $Q$-spectrum). We say that a graph $G$ is determined by its spectrum (resp., $L$-spectrum, $Q$-spectrum) or simply $G$ is $D S$ (resp., $D L S, D Q S$ ) if there is no non-isomorphic graph cospectral to $G$.

One of the interesting problems in spectral graph theory is to characterize graphs which are determined by their spectra. This question was raised by Günthard and Primas [12] with motivations from Hückel theory. In [25, 26], Dam and Haemers gave a survey of (partial) answers to the posed question. In literature,

\footnotetext{
*Received by the editors on April 6, 2018. Accepted for publication on May 28, 2020. Handling Editor: Bryan L. Shader. Corresponding Author: Kinkar Chandra Das.

${ }^{\dagger}$ Department of Studies in Mathematics, University of Mysore Manasagangothri, Mysuru - 570 006, India (c_adiga@hotmail.com).

${ }^{\ddagger}$ Department of Mathematics, Sungkyunkwan University, Suwon 16419, Republic of Korea (kinkardas2003@googlemail.com). The second author was supported by the Sungkyun research fund, Sungkyunkwan University, 2017, and National Research Foundation of the Korean government with grant no. 2017R1D1A1B03028642.

$\S$ Department of Mathematics, Vidyavardhaka College of Engineering, Mysuru - 570 002, India (ranmsc08@yahoo.co.in).
} 
there are several papers addressing the problem of characterizing graphs which are $D S, D L S$ and $D Q S$. For some recent papers on this topic, see [8, 14, 16, 22, 24].

The distance between the vertices $v_{i}$ and $v_{j}$ of $G$ is the length of a shortest path between them. It is denoted by $d\left(v_{i}, v_{j}\right)$. The maximum of all distances between any pair of vertices of $G$ is the diameter of $G$. The distance matrix $D(G)$ of a connected graph $G$ is the real symmetric matrix of order $n$ with $(i, j)$-entry equal to $d\left(v_{i}, v_{j}\right)$. The distance spectrum or $D$-spectrum of $G$ is the spectrum of $D(G)$. Two connected graphs are $D$-cospectral if they have same $D$-spectrum. A connected graph $G$ is $D D S$ if there is no non-isomorphic graph $D$-cospectral to $G$. In [18], Lin et al. showed that the complete graph, the complete bipartite graph and the complete split graph are $D D S$. Further, they conjectured that the complete multipartite graph is $D D S$ and this conjecture was confirmed by Jin and Zhang in [17]. In [28], Xue et al. proved that the path graph and double star graph is determined by their distance spectrum. In [8], Das and Liu proved that the kite graph $K i_{n, n-1}$ (for definition, see [8]) is $D D S$.

In [4], Cámara and Haemers proved that the graph $K_{n} \backslash K_{l, m}$ is $D S$. In [29], Zhou and Bu showed that if $G$ is a disconnected $D L S$ graph, then the join graph $G \vee K_{r}$ is $D L S$. From this, it follows that the graph $K_{n} \backslash K_{l, m}$ with $m>1$ and $\frac{l}{m}>\frac{5}{3}$ is $D L S$, since $K_{l} \cup K_{m}$ with $m>1$ and $\frac{l}{m}>\frac{5}{3}$ is $D L S$, see [1]. Motivated by these results, in Section 3 of this paper, we show that the graph $K_{n} \backslash K_{1, m}$ with $m \geq 4$ is determined by its signless Laplacian spectrum and we also prove that the graph $K_{n} \backslash K_{l, m}$ is determined by its distance spectrum. Recently, the signless Laplacian spectral determination of the join of graphs has been studied, for example, we have the following joins which are $D Q S$.

1. $K_{1} \vee\left(c K_{2} \cup(n-2 c-1) K_{1}\right)$ with $n \geq 2 c+1$ and $c \geq 0$ [20].

2. $K_{1} \vee C_{n}$, where $C_{n}$ is the cycle with $n$ vertices [19].

3. $G \vee K_{1}$, where $G$ is an $r$-regular graph on $n$ vertices and $r=1$ or $n-2$ or 2 with $n \geq 11$, and $G \vee K_{1}$, where $G$ is an ( $\left.n-3\right)$-regular with $\bar{G}$ having no triangles [3].

4. $G \vee K_{2}$, where $G$ is an $r$-regular graph on $n$ vertices and $r=1$ or $n-2$, and $G \vee K_{2}$, where $G$ is an $(n-3)$-regular with $\bar{G}$ having no triangles [27].

5. $G \vee K_{m}$, where $G$ is an $(n-2)$-regular graph and $\bar{K}_{n} \vee K_{2}$ for $n \neq 3$ [21].

6. The complete split graph $\bar{K}_{n} \vee K_{m}$ for $n \neq 3[7]$.

Motivated by these results, in Section 4, we prove that the join graph $G \vee K_{n}$ is determined by its signless Laplacian spectrum, where $G$ is a 1-regular graph. This result extends the following known theorem.

TheOREM 1.1. ([3, 27]) Let $G$ be a 1-regular graph. Then for $r=1,2, G \vee K_{r}$ is determined by its signless Laplacian spectrum.

2. Some preliminary results. In this section, we give some necessary theorems and lemmas required to prove our main results. We denote the eigenvalues of a Hermitian matrix $M$ of order $m$ by $\theta_{1}(M) \geq$ $\theta_{2}(M) \geq \cdots \geq \theta_{m}(M)$ and also, let $\gamma_{1}(G) \geq \gamma_{2}(G) \geq \cdots \geq \gamma_{n}(G)$ and $\eta_{1}(G) \geq \eta_{2}(G) \geq \cdots \geq \eta_{n}(G)$ be the signless Laplacian eigenvalues and distance eigenvalues of $G$, respectively.

TheOREm 2.1. ([15]) Let $M$ be a Hermitian matrix of order $n$.

(i) If $M_{k}$ is a principal submatrix of $M$ of order $k$ with $1 \leq k \leq n$, then for $1 \leq i \leq k, \theta_{n-k+i}(M) \leq$ $\theta_{i}\left(M_{k}\right) \leq \theta_{i}(M)$.

(ii) If $M=N+P$, where $N$ and $P$ are Hermitian matrices of order $n$. Then for $1 \leq i, j \leq n$, we have:

(a) $\theta_{i}(N)+\theta_{j}(P) \leq \theta_{i+j-n}(M)(i+j>n)$;

(b) $\theta_{i+j-1}(M) \leq \theta_{i}(N)+\theta_{j}(P)(i+j-1 \leq n)$. 
A connected bipartite graph $G$ with vertex partition sets $U$ and $V$ is called as balanced if the cardinalities of $U$ and $V$ are same.

THEOREM 2.2. ([5, 6, 8, 11]) Let $G$ be a connected graph of order $n$.

(i) We have $\gamma_{2}(G) \leq n-2$, for $n \geq 2$. Moreover, $\gamma_{k+1}(G)=n-2(1 \leq k \leq n-1)$ if and only if $\bar{G}$ has either $k$ balanced bipartite components or $k+1$ bipartite components.

(ii) $d_{n-1}(G) \geq \gamma_{n-1}(G)-1$. Furthermore, if the equality holds, then $d_{n-1}(G)=d_{n}(G)$.

(iii) If $G$ is a bipartite graph, then the $Q$-spectrum of $G$ is equal to its L-spectrum.

(iv) The largest Laplacian eigenvalue of $G$ is at most $n$ and $\gamma_{1}(G) \leq 2 d_{1}(G)$.

(v) The multiplicity of 0 as an eigenvalue pertaining to $Q(G)$ is the number of connected bipartite components of $G$.

LEMma 2.3. Let $l+m \leq n-1$. Then the signless Laplacian spectrum of $K_{n} \backslash K_{l, m}$ consists of $n-2$ of multiplicity $n-l-m, n-m-2$ of multiplicity $l-1, n-l-2$ of multiplicity $m-1$, and the two roots of the quadratic polynomial $x^{2}+(l+m-3 n+4) x+2 n^{2}-(2 l+2 m+6) n+(4 m+2) l+2 m+4$.

Proof. The signless Laplacian matrix $Q$ of $K_{n} \backslash K_{l, m}$ can be written as follows:

$$
\left[\begin{array}{ccc}
J_{n-m-l}+(n-2) I_{n-m-l} & J & J \\
J & J_{l}+(n-m-2) I_{l} & 0 \\
J & 0 & J_{m}+(n-l-2) I_{m}
\end{array}\right],
$$

where $J$ is a matrix whose all entries are 1 and $I_{m}$ is the identity matrix of order $m$. From the above matrix, we see that the matrices $Q-(n-2) I_{n}, Q-(n-m-2) I_{n}$ and $Q-(n-l-2) I_{n}$ have rank at most $l+m+1$, $n-l+1$ and $n-m+1$, respectively. This implies that the matrices $Q-(n-2) I_{n}, Q-(n-m-2) I_{n}$ and $Q-(n-l-2) I_{n}$ have nullity at least $n-l-m-1, l-1$ and $m-1$, respectively. Thus, the spectrum of $Q$ consists of $n-2$ with multiplicity $n-l-m-1, n-m-2$ with multiplicity $l-1$ and $n-l-2$ with multiplicity $m-1$. Now observe that the given partition of $Q$ is equitable (see [2]) with the quotient matrix

$$
Q_{1}=\left[\begin{array}{ccc}
2 n-m-l-2 & l & m \\
n-m-l & n-m+l-2 & 0 \\
n-m-l & 0 & n-l+m-2
\end{array}\right] .
$$

The spectrum of $Q_{1}$ consists of $n-2$ and the two roots of the polynomial $x^{2}+(l+m-3 n+4) x+2 n^{2}-$ $(2 l+2 m+6) n+(4 m+2) l+2 m+4$. As the spectrum of $Q_{1}$ is contained in the spectrum of $Q$, see [2], we are done.

The following lemma gives the $D$-spectrum of $K_{n} \backslash K_{l, m}$. As the proof of the lemma is in similar lines of the above lemma, we omit the details.

LEMMA 2.4. The distance spectrum of $K_{n} \backslash K_{l, m}$ consists of -1 with multiplicity $n-3$ and the three roots of the polynomial $x^{3}-(n-3) x^{2}-(3 l m+2 n-3) x-m l^{2}-m(m-n+3) l-n+1$.

LEMma 2.5. ([10]) For $i=1,2$, let $G_{i}$ be an $r_{i}$-regular graph on $n_{i}$ vertices. Then the $Q$-spectrum of $G_{1} \vee G_{2}$ consists of $\gamma_{j}\left(G_{1}\right)+n_{2}\left(j=2,3, \ldots, n_{1}\right), \gamma_{j}\left(G_{2}\right)+n_{1},\left(j=2,3, \ldots, n_{2}\right)$ and the two roots of the quadratic polynomial $x^{2}-\left(2\left(r_{1}+r_{2}\right)+\left(n_{1}+n_{2}\right)\right) x+2\left(2 r_{1} r_{2}+r_{1} n_{1}+r_{2} n_{2}\right)$.

LEMMA 2.6. Let $Q_{1}=J_{n-m+1}+(n-2) I_{n-m+1}$ be a square matrix of order $n-m+1$ with $n>m+1$. Then $n-2$ is an eigenvalue of multiplicity $n-m$ and $2 n-m-1$ is the remaining eigenvalue of $Q_{1}$.

Proof. Let $\mathbf{x}=\left(x_{1}, x_{2}, \ldots, x_{n}\right)^{T}$ be an eigenvector corresponding to the eigenvalue $\theta$ of $Q_{1}$. Then 
$Q_{1} \mathbf{x}=\theta \mathbf{x}$. One can easily see that the eigenvalue $n-2$ with corresponding eigenvectors

$$
(\underbrace{1,-1}_{2}, 0, \ldots, 0)^{T},(\underbrace{1,0,-1}_{3}, 0, \ldots, 0)^{T}, \ldots, \text { and }(\underbrace{1,0, \ldots, 0,-1}_{n-m+1})^{T}
$$

satisfy the above relation. Since these $n-m$ eigenvectors are linearly independent, $n-2$ is an eigenvalue with multiplicity at least $n-m$. Since $\sum_{i=1}^{n-m+1} \theta_{i}\left(Q_{1}\right)=(n-1)(n-m+1), 2 n-m-1$ is the remaining eigenvalue.

Corollary 2.7. Let $Q_{1}=J_{n-m+1}+(n-2) I_{n-m+1}$ be a square matrix of order $n-m+1$ with $n>m+1$. Then $\theta_{n-m+1}\left(Q_{1}\right)=n-2$.

Proof. Since $n>m+1$, by Lemma 2.6, we get the required result.

Lemma 2.8. Let $G$ be a graph of order $2 \alpha$, where the spectrum of the signless Laplacian of $G$ is

$$
Q_{S}(G)=(4 \alpha-4, \underbrace{2 \alpha-2, \ldots, 2 \alpha-2}_{\alpha}, \underbrace{2 \alpha-4, \ldots, 2 \alpha-4}_{\alpha-1}) .
$$

Then $G$ is regular of degree $(2 \alpha-2)$.

Proof. From the signless Laplacian spectrum of $G$, one can easily see that

$$
\sum_{i=1}^{2 \alpha} d_{i}=\sum_{i=1}^{2 \alpha} \gamma_{i}=4 \alpha(\alpha-1) \quad \text { and } \quad \sum_{i=1}^{2 \alpha} d_{i}^{2}=\sum_{i=1}^{2 \alpha} \gamma_{i}^{2}-\sum_{i=1}^{2 \alpha} \gamma_{i}=8 \alpha(\alpha-1)^{2} .
$$

Thus, we have

$$
\sum_{i=1}^{2 \alpha}\left(d_{i}-2(\alpha-1)\right)^{2}=0, \quad \text { that is, } d_{i}=2 \alpha-2, \quad i=1,2, \ldots, 2 \alpha .
$$

Hence, $G$ is regular of degree $(2 \alpha-2)$.

Let $\lambda_{1}(G)$ denote the spectral radius of the graph $G$ and also let $L_{G}$ be the line graph of $G$. The following result was obtained in $[9,13]$ :

Lemma 2.9. $([9,13])$ Let $G$ be a connected graph of order $n$. Then $\gamma_{1}(G)=2+\lambda_{1}\left(L_{G}\right)$.

Corollary 2.10. Let $G$ be a connected graph of order $n$ with $m$ edges. Then $\gamma_{1}(G) \leq m+1$ with equality holding if and only if $L_{G} \cong K_{m}$.

Proof. It is well known that the adjacency spectral radius $\lambda_{1}(G) \leq n-1$ with equality holding if and only if $G \cong K_{n}$. Then by Lemma 2.9 , we have $\gamma_{1}(G) \leq m+1$ as $m$ is the number of edges in $G$. Moreover, the equality holds if and only if $L_{G} \cong K_{m}$.

3. Signless Laplacian (distance) spectral characterization of $K_{n} \backslash K_{l, m}$. Let $\lambda_{1}(G) \geq \lambda_{2}(G) \geq$ $\cdots \geq \lambda_{n}(G)$ be the spectrum of $G$. The following theorem gives a general form of Theorem 2.2 (ii).

THEOREM 3.1. Let $G$ be a graph with $n$ vertices and let $H_{i}$ be the graph induced by the vertices $v_{n-i+1}, v_{n-i+2}, \ldots, v_{n}$ of $G$, where $2 \leq i \leq n$. Then

$$
\gamma_{n-i+1}(G)-\lambda_{1}\left(H_{i}\right) \leq d_{n-i+1}(G) .
$$


Proof. Let $A\left(H_{i}\right)$ be the adjacency matrix of $H_{i}$ with its rows and columns indexed by $v_{n-i+1}, v_{n-i+2}$, $\ldots, v_{n}$. Let $Q_{i}=D_{i}+A\left(H_{i}\right)$, where $D_{i}=\operatorname{diag}\left(d_{n-i+1}(G), d_{n-i+2}(G), \ldots, d_{n}(G)\right)$. Then clearly, $Q_{i}$ is a principal submatrix of $Q(G)$ and so by Theorem 2.1 (i), we have $\theta_{1}\left(Q_{i}\right) \geq \gamma_{n-i+1}(G)$. Hence, $\gamma_{n-i+1}(G) \leq$ $\theta_{1}\left(Q_{i}\right) \leq d_{n-i+1}(G)+\lambda_{1}\left(H_{i}\right)$ by Theorem 2.1 (ii).

In literature, complete graph $K_{n}$ with some edges deleted is studied for spectral determination. In the following theorem, it is shown that if the deleted edges form a star graph with at least 4 edges, then it is determined by its signless Laplacian spectrum.

THEOREM 3.2. If $n>m+1$ and $m \geq 4$, then $K_{n} \backslash K_{1, m}$ is $D Q S$.

Proof. Let $G$ be a graph $Q$-cospectral with $K_{n} \backslash K_{1, m}$. Then by Lemma 2.3, the $Q$-spectrum of $G$ is

$$
\begin{aligned}
& \left(3 n-m-5 \pm \sqrt{m^{2}+(2 n-14) m+(n+1)^{2}}\right) / 2, \\
& n-2 \text { with multiplicity } n-m-1 \\
& n-3 \text { with multiplicity } m-1
\end{aligned}
$$

This implies

$$
\left.\begin{array}{l}
2|E(G)|=\sum_{i=1}^{n} d_{i}(G)=\sum_{i=1}^{n} \gamma_{i}(G)=n^{2}-2 m-n, \\
\sum_{i=1}^{n} d_{i}(G)\left(d_{i}(G)+1\right)=\sum_{i=1}^{n} \gamma_{i}^{2}(G)=n^{3}-n^{2}+m^{2}-(4 n-3) m .
\end{array}\right\} .
$$

Since $n>m+1$, from (3.1), one can easily see that

$$
\gamma_{1}(G)=\left(3 n-m-5+\sqrt{m^{2}+(2 n-14) m+(n+1)^{2}}\right) / 2>2(n-2),
$$

and $\gamma_{1}(G) \leq 2 d_{1}(G)$ by Theorem 2.2 (iv). It follows that $d_{1}(G)=n-1$, and hence, $G$ is connected. Since

$$
\left(3 n-m-5-\sqrt{m^{2}+(2 n-14) m+(n+1)^{2}}\right) / 2<n-3
$$

by Theorem 2.2 (ii) and (3.1), we have $d_{n-1}(G) \geq n-4$. Furthermore, if $d_{n-1}(G)=n-4$, then $d_{n-1}(G)=$ $d_{n}(G)$. Let $v_{n}$ be a vertex of degree $d_{n}(G)$ and let $n_{i}$ be the number of vertices in $V(G) \backslash\left\{v_{n}\right\}$ of degree $n-i$, $i=1,2,3,4$. Suppose $d_{n-m+1}(G)=n-1$. Then $Q_{1}=J_{n-m+1}+(n-2) I_{n-m+1}$ is a principal submatrix of $Q(G)$ and so by Theorem 2.1 (i) with Corollary 2.7, we have $\gamma_{n-m+1}(G) \geq \theta_{n-m+1}\left(Q_{1}\right)=n-2$. Since $\gamma_{n-m+1}(G)=n-3$, this is a contradiction. Thus, $1 \leq n_{1} \leq n-m$.

From (3.2), we have

$$
\begin{gathered}
n_{4}+n_{3}+n_{2}+n_{1}=n-1, \\
(n-4) n_{4}+(n-3) n_{3}+(n-2) n_{2}+(n-1) n_{1}=n^{2}-2 m-n-d_{n}(G), \\
(n-4)^{2} n_{4}+(n-3)^{2} n_{3}+(n-2)^{2} n_{2}+(n-1)^{2} n_{1}=n^{3}-2 n^{2}-(4 m-1) n+m^{2}+5 m-d_{n}^{2}(G) .
\end{gathered}
$$


Suppose $d_{n-1}(G)=n-4$. Then $d_{n}(G)=d_{n-1}(G)=n-4$ and by equations (3.3)-(3.5), we have

$$
\begin{gathered}
n_{3}+2 n_{2}+3 n_{1}=3 n-2 m \\
(n-3) n_{3}+2(n-2) n_{2}+3(n-1) n_{1}=3 n^{2}-3 n+m^{2}-(2 n+3) m .
\end{gathered}
$$

Eliminating $n_{3}$ from (3.6) and (3.7), we have

$$
n_{2}=(1 / 2) m^{2}-(9 / 2) m+3 n-3 n_{1} .
$$

Substituting (3.8) in (3.6), we have

$$
n_{3}=-m^{2}+7 m-3 n+3 n_{1} \text {. }
$$

Since $m \geq 4$ and $n_{1} \leq n-m$, from (3.9), we have $n_{3}=0$ and $n_{1}=n-4$. These results with (3.3) and (3.4), we get $n_{2}+n_{4}=3$ and $n_{4}=m-3$. Therefore, by (3.8), it follows that $n_{2}=2$ and $n_{4}=1$. This is impossible because if $n_{1}=n-4, n_{4}=1$ and $d_{n}(G)=n-4$, then we must have $n_{2}=0$. Hence, $d_{n-1}(G) \geq n-3$ and $n_{4}=0$ holds in equations (3.3)-(3.5). Now from (3.3) and (3.4), we have

$$
n_{2}+2 n_{1}=3 n-2 m-d_{n}(G)-3 .
$$

Eliminating $n_{3}$ from (3.3) and (3.5), we have

$$
(2 n-5) n_{2}+4(n-2) n_{1}=5 n^{2}+m^{2}-4 m n+5 m-14 n+9-d_{n}^{2}(G) .
$$

Solving equations (3.10) and (3.11) for $n_{1}$, we have

$$
n_{1}=(1 / 2) m^{2}-(1 / 2) n^{2}+(n-5 / 2) d_{n}(G)-(1 / 2) d_{n}^{2}(G)-(5 / 2) m+(7 / 2) n-3 .
$$

If $d_{n}(G) \geq n-2$, then by (3.12), we have $n_{1} \geq n-3$, a contradiction, since $n_{1} \leq n-m$ and $m \geq 4$. Thus, $1 \leq d_{n}(G) \leq n-3$.

Now, define

$$
\phi(x)=(1 / 2) m^{2}-(1 / 2) n^{2}+n x-(1 / 2) x^{2}-(5 / 2) m+(7 / 2) n-(5 / 2) x-3 .
$$

Then

$$
\phi^{\prime}(x)=n-x-5 / 2>0,1 \leq x \leq n-3 .
$$

Hence, $\phi(x)$ is an increasing function on $1 \leq x \leq n-3$. If $n-m \leq d_{n}(G) \leq n-3$, then $\phi(n-m)=n-3 \leq$ $n_{1} \leq n-m$, a contradiction, since $m \geq 4$. Thus, $d_{n}(G) \leq n-m-1$. Since $|E(G)|=\frac{1}{2}(n-2)(n-1)+n-m-1$, we have

$$
\begin{aligned}
(n-2)(n-1)+2(n-m-1) & =2|E(G)| \\
& \leq d_{n}(G)+d_{n}(G)(n-1)+\left(n-d_{n}(G)-1\right)(n-2) \\
& =(n-1)(n-2)+2 d_{n}(G) .
\end{aligned}
$$

If $d_{n}(G) \leq n-m-2$, then from (3.13), we get a contradiction. Thus, we have $d_{n}(G)=n-m-1$. Again since $|E(G)|=\frac{1}{2}(n-2)(n-1)+n-m-1$, we have $G \backslash\left\{v_{n}\right\} \cong K_{n-1}$. Hence, $G \cong K_{n} \backslash K_{1, m}$. 
Electronic Journal of Linear Algebra, ISSN 1081-3810

A publication of the International Linear Algebra Society

Volume 36, pp. 461-472, July 2020.

THEOREM 3.3. Let $G$ be a connected graph on $n$ vertices with diameter 2 and having a distance eigenvalue -1 with multiplicity $n-3$. Then $G \cong K_{n} \backslash K_{l, m}$, where $l, m \geq 1$ and $l+m \leq n-1$.

Proof. Since -1 is a distance eigenvalue of $G$ with multiplicity $n-3$, it follows that the symmetric matrix $D(G)+I$ is of rank 3. Thus, we can assume that

$$
D(G)+I=\left[\begin{array}{cc}
D_{1} & X \\
X^{T} & D_{2}
\end{array}\right]
$$

where $D_{1}$ is a nonsingular matrix of order 3. Since the nullity of $D(G)+I$ is $n-3$ and $D_{1}$ is a nonsingular matrix of order 3 , we have $D_{2}=X^{T} D_{1}^{-1} X$. Thus, $x^{T} D_{1}^{-1} x=1$ for each column $x$ of $X$, since $D_{2}$ is a matrix with 1 as its diagonal entries. Now as $D_{1}-I$ is a principal submatrix of $D(G)$, the distance matrix of $G$ with diameter 2 and $\operatorname{rank}\left(D_{1}\right)=3$, we have

$$
D_{1}=\left[\begin{array}{lll}
1 & 2 & 2 \\
2 & 1 & 2 \\
2 & 2 & 1
\end{array}\right] \quad \text { or } \quad D_{1}=\left[\begin{array}{lll}
1 & 1 & 1 \\
1 & 1 & 2 \\
1 & 2 & 1
\end{array}\right]
$$

In the first case,

$$
D_{1}^{-1}=(1 / 5)\left[\begin{array}{ccc}
-3 & 2 & 2 \\
2 & -3 & 2 \\
2 & 2 & -3
\end{array}\right]
$$

and it is easy to see that the only possible columns $x$ of $X$ satisfying $1=x^{T} D_{1}^{-1} x$ are $[1,2,2]^{T},[2,1,2]^{T}$ and $[2,2,1]^{T}$. In the second case,

$$
D_{1}^{-1}=\left[\begin{array}{ccc}
3 & -1 & -1 \\
-1 & 0 & 1 \\
-1 & 1 & 0
\end{array}\right]
$$

and so $x^{T} D_{1}^{-1} x=1$ implies that $x$ is one of the three vectors $[1,1,1]^{T},[1,1,2]^{T}$ and $[1,2,1]^{T}$. Thus, these two cases leads to the following two possibilities for $D(G)+I$ :

$$
D(G)+I=\left[\begin{array}{ccc}
J_{k} & 2 J & 2 J \\
2 J & J_{l} & 2 J \\
2 J & 2 J & J_{m}
\end{array}\right] \quad \text { or } \quad\left[\begin{array}{ccc}
J_{k} & J & J \\
J & J_{l} & 2 J \\
J & 2 J & J_{m}
\end{array}\right] \text {. }
$$

The first possibility is impossible because $G$ is a connected graph. Thus, we must have

$$
D(G)+I=\left[\begin{array}{ccc}
J_{k} & J & J \\
J & J_{l} & 2 J \\
J & 2 J & J_{m}
\end{array}\right], \quad \text { i.e., } \quad G \cong K_{n} \backslash K_{l, m} .
$$

THEOREM 3.4. The graph $K_{n} \backslash K_{l, m}$ is $D D S$.

Proof. Let $G$ be a graph $D$-cospectral with $K_{n} \backslash K_{l, m}$. If the diameter of $G$ is at least 3 , then $G$ has $P_{4}$, the path of length 3 as its induced subgraph and $D\left(P_{4}\right)$ as a principal submatrix of $D(G)$. Therefore, by Theorem 2.1 (i), $\eta_{2}(G) \geq-0.586$ and $\eta_{3}\left(P_{4}\right) \cong-1.162 \geq \eta_{n-1}(G)$, which is a contradiction to Lemma 2.4 . Thus, the diameter of $G$ is at most 2. Clearly $G \nsubseteq K_{n}$ and so $G$ is of diameter 2 . Hence, by Theorem 3.3 , $G \cong K_{n} \backslash K_{l_{1}, m_{1}}$. Since $G$ and $K_{n} \backslash K_{l, m}$ have same $Q$-spectrum, from Lemma 2.4, we have $l m=l_{1} m_{1}$ and $l+m=l_{1}+m_{1}$. Solving these equations, we get $l=l_{1}$ and $m=m_{1}$. Thus, $G \cong K_{n} \backslash K_{l, m}$. 


\section{Signless Laplacian spectral characterization of the join graph $\alpha K_{2} \vee K_{n-2 \alpha}$.}

In this section, we prove that the multicone graph $\alpha K_{2} \vee K_{n-2 \alpha}$ is determined by its signless Laplacian spectrum which complement the works of $\mathrm{Bu}$ and $\mathrm{Zhou}$ in [3], and $\mathrm{Xu}$ and $\mathrm{He}$ in [27].

THEOREM 4.1. Let $n$ and $\alpha$ be positive integers with $n-2 \alpha \geq 1$. Then the graph $\alpha K_{2} \vee K_{n-2 \alpha}$ is DQS.

Proof. Let $G \cong \alpha K_{2} \vee K_{n-2 \alpha}(n-2 \alpha \geq 1)$. When $\alpha=1, G$ is a complete graph and so $G$ is $Q D S$. For $n-2 \alpha=1$, by Theorem 1.1, $G \cong \alpha K_{2} \vee K_{1}$ is determined by its $Q$-spectrum. Otherwise, $\alpha \geq 2$ and $n-2 \alpha \geq 2$. Let $H$ be a graph $Q$-cospectral with $G$. Then by Lemma 2.5, the $Q$-spectrum of $H$ is

$$
\begin{aligned}
& \gamma_{1}(H), \gamma_{n}(H)=\left(3 n-4 \alpha \pm \sqrt{n^{2}+8 n(\alpha-1)-16 \alpha^{2}+16}\right) / 2 \\
& \gamma_{2}(H)=\gamma_{3}(H)=\cdots=\gamma_{n-2 \alpha}(H)=n-2 \\
& \gamma_{n-2 \alpha+1}(H)=\gamma_{n-2 \alpha+2}(H)=\cdots=\gamma_{n-\alpha-1}(H)=n-2 \alpha+2 \\
& \gamma_{n-\alpha}(H)=\gamma_{n-\alpha+1}(H)=\cdots=\gamma_{n-1}(H)=n-2 \alpha
\end{aligned}
$$

Let $\bar{H}$ be the complement graph of $H$. From Theorem 2.1 (ii) and (4.14), we have

$$
\begin{aligned}
& n-2 \leq \gamma_{n-2 \alpha}(H)+\gamma_{2 \alpha+2}(\bar{H}) \leq \theta_{2}(Q(H)+Q(\bar{H}))=n-2, \\
& n-2=\theta_{n}(Q(H)+Q(\bar{H})) \leq \gamma_{n-2 \alpha+1}(H)+\gamma_{2 \alpha}(\bar{H})=n-2 \alpha+2+\gamma_{2 \alpha}(\bar{H}), \\
& n-2 \alpha+2+\gamma_{\alpha+3}(\bar{H})=\gamma_{n-\alpha-1}(H)+\gamma_{\alpha+3}(\bar{H}) \leq \theta_{2}(Q(H)+Q(\bar{H}))=n-2, \\
& n-2=\theta_{n}(Q(H)+Q(\bar{H})) \leq \gamma_{n-\alpha}(H)+\gamma_{\alpha+1}(\bar{H})=n-2 \alpha+\gamma_{\alpha+1}(\bar{H}) \\
& \text { and } \\
& n-2 \alpha+\gamma_{3}(\bar{H})=\gamma_{n-1}(H)+\gamma_{3}(\bar{H}) \leq \theta_{2}(Q(H)+Q(\bar{H}))=n-2 .
\end{aligned}
$$

Thus,

$$
\left.\begin{array}{l}
\gamma_{3}(\bar{H})=\gamma_{4}(\bar{H})=\cdots=\gamma_{\alpha+1}(\bar{H})=2 \alpha-2, \\
\gamma_{\alpha+3}(\bar{H})=\gamma_{\alpha+4}(\bar{H})=\cdots=\gamma_{2 \alpha}(\bar{H})=2 \alpha-4, \\
\gamma_{2 \alpha+2}(\bar{H})=\gamma_{2 \alpha+3}(\bar{H})=\cdots=\gamma_{n}(\bar{H})=0 .
\end{array}\right\} .
$$

Since $G$ and $H$ are $Q$-cospectral, it is easy to see that

$$
\begin{aligned}
& \sum_{i=1}^{n} \gamma_{i}(\bar{H})=2|E(\bar{H})|=\sum_{i=1}^{n} d_{i}(\bar{H})=\sum_{i=1}^{n} d_{i}(\bar{G})=4 \alpha(\alpha-1), \\
& \sum_{i=1}^{n} \gamma_{i}^{2}(\bar{H})=\sum_{i=1}^{n} d_{i}(\bar{H})\left(d_{i}(\bar{H})+1\right)=\sum_{i=1}^{n} d_{i}(\bar{G})\left(d_{i}(\bar{G})+1\right)=4 \alpha(\alpha-1)(2 \alpha-1) .
\end{aligned}
$$

If $\alpha=2$, then from (4.16), $|E(\bar{H})|=4$. Thus, we have the following possibilities for $\bar{H}$.

$$
\bar{H} \cong\left\{\begin{array}{l}
H_{1} \cup(n-5) K_{1}, \quad H_{1} \text { is a tree on } 5 \text { vertices, } \\
H_{1} \cup(n-4) K_{1}, \quad H_{1} \text { is a connected graph with } 4 \text { vertices and } 4 \text { edges, } \\
K_{3} \cup K_{2} \cup(n-5) K_{1} \text { or } P_{4} \cup K_{2} \cup(n-6) K_{1} \text { or } S_{1,3} \cup K_{2} \cup(n-6) K_{1}, \text { or } \\
P_{3} \cup P_{3} \cup(n-6) K_{1} \text { or } P_{3} \cup K_{2} \cup K_{2} \cup(n-7) K_{1} \text { or } 4 K_{2} \cup(n-8) K_{1} .
\end{array}\right.
$$


By Sage [23], using the $Q$-spectrum of connected graphs with at most 5 vertices, one can easily check that all these cases for $\bar{H}$, except $\bar{H} \cong K_{2,2} \cup(n-4) K_{1}$ contradicts either (4.15) or (4.17). Thus, $\bar{H} \cong K_{2,2} \cup(n-4) K_{1}$, and hence, the theorem is true for $1 \leq \alpha \leq 2$. Now let $\alpha \geq 3$. The following claim is important for our proof.

Claim: $\bar{H}$ has at least one connected component $H_{1}$ with $\gamma_{2}\left(H_{1}\right) \geq 2 \alpha-2$.

Proof of Claim: Let $\bar{H}=H_{1} \cup H_{2} \cup \cdots \cup H_{k} \cup r K_{1}$, where $H_{i}(1 \leq i \leq k)$ is $i$-th connected component of order $n_{i}$ in $\bar{H}\left(r=n-n_{1}-n_{2}-\cdots-n_{k}\right)$. Then $\gamma(\bar{H})=\gamma\left(H_{1}\right) \cup \gamma\left(H_{2}\right) \cup \cdots \cup \gamma\left(H_{k}\right) \cup\{\underbrace{0, \ldots, 0}_{r}\}$, where $\gamma\left(H_{i}\right)$ denotes the signless Laplacian spectrum of $H_{i}$. Suppose to the contrary that $\gamma_{2}\left(H_{i}\right)<2 \alpha-2$ for all $i$, $1 \leq i \leq k$. Since $\gamma_{1}(\bar{H}) \geq \gamma_{2}(\bar{H}) \geq \gamma_{3}(\bar{H})=\gamma_{4}(\bar{H})=\cdots=\gamma_{\alpha+1}(\bar{H})=2 \alpha-2$, there exists $\alpha+1$ connected components $H_{i_{1}}, H_{i_{2}}, \ldots, H_{i_{\alpha+1}}$ such that

$$
\gamma_{1}(\bar{H})=\gamma_{1}\left(H_{i_{1}}\right), \quad \gamma_{2}(\bar{H})=\gamma_{1}\left(H_{i_{2}}\right), \quad \ldots, \quad \gamma_{\alpha+1}(\bar{H})=\gamma_{1}\left(H_{i_{\alpha+1}}\right) .
$$

Since $\gamma_{2}\left(H_{i_{j}}\right)<2 \alpha-2(1 \leq j \leq \alpha+1)$ and $\bar{H}$ has at most $\alpha$ non-zero signless Laplacian eigenvalues which are strictly less than $2 \alpha-2$, there exist at least one connected component say $H_{i_{j}}$ such that $\gamma_{2}\left(H_{i_{j}}\right)=0$. Therefore, $H_{i_{j}}$ is of order 2, since $\gamma_{2}\left(H_{i_{j}}\right)=0$ and 0 can be a signless Laplacian eigenvalue of a connected graph with multiplicity at most 1 . So $H_{i_{j}} \cong K_{2}$. This implies that $2=\gamma_{1}\left(H_{i_{j}}\right) \geq 2 \alpha-2>2$ as $\alpha \geq 3$. This is a contradiction, and the claim is proven.

Let $H_{1}$ be a connected component of $\bar{H}$ with $\gamma_{2}\left(H_{1}\right) \geq 2 \alpha-2$, then from Theorem 2.2 (i), $2 \alpha-2 \leq$ $\gamma_{2}\left(H_{1}\right) \leq\left|V\left(H_{1}\right)\right|-2$, thus $\left|V\left(H_{1}\right)\right| \geq 2 \alpha$. From (4.15) and Theorem $2.2(\mathrm{v})$, it follows that the number of bipartite components of $\bar{H}$ is either $n-2 \alpha$ or $n-2 \alpha-1$. First we assume that the number of bipartite components of $\bar{H}$ is exactly $n-2 \alpha$. Then we have $\gamma_{2 \alpha+1}(\bar{H})=0$. Since $\left|V\left(H_{1}\right)\right| \geq 2 \alpha$, then we have the following possibilities for $\bar{H}$.

$$
\bar{H} \cong\left\{\begin{array}{l}
H_{1} \cup(n-2 \alpha) K_{1}, H_{1} \text { is a non-bipartite graph of order } 2 \alpha, \\
H_{1} \cup(n-2 \alpha-2) K_{1} \cup K_{2}, H_{1} \text { is a bipartite graph of order } 2 \alpha, \text { or } \\
H_{1} \cup(n-2 \alpha-1) K_{1}, H_{1} \text { is a bipartite graph of order } 2 \alpha+1 .
\end{array}\right.
$$

Case $I: \bar{H} \cong H_{1} \cup(n-2 \alpha) K_{1}, H_{1}$ is a non-bipartite graph of order $2 \alpha$. Since $\left|V\left(H_{1}\right)\right|=2 \alpha$ and $\gamma_{2}(\bar{H}) \geq 2 \alpha-2$, by Theorem $2.2(\mathrm{i})$, we have $\gamma_{2}(\bar{H})=2 \alpha-2$. From (4.15), (4.16) and (4.17), we get

$$
\gamma_{1}(\bar{H})+\gamma_{\alpha+2}(\bar{H})=6 \alpha-8 \quad \text { and } \quad \gamma_{1}^{2}(\bar{H})+\gamma_{\alpha+2}^{2}(\bar{H})=20 \alpha^{2}-48 \alpha+32
$$

Solving these equations, we obtain $\gamma_{1}(\bar{H})=4(\alpha-1)$ and $\gamma_{\alpha+2}(\bar{H})=2 \alpha-4$. By Lemma 2.8, $H_{1}$ is a $(2 \alpha-2)$-regular graph on $2 \alpha$ vertices. Therefore, $\bar{H}_{1} \cong \alpha K_{2}$, and hence, $H \cong G$.

Case II: $\bar{H} \cong H_{1} \cup(n-2 \alpha-2) K_{1} \cup K_{2}, H_{1}$ is a bipartite graph of order $2 \alpha$. Then we have $\gamma_{2 \alpha}(\bar{H})=$ $2 \alpha-4 \leq 2$, that is $\alpha \leq 3$, and hence, $\alpha=3$. From (4.16), $H_{1}$ is a bipartite graph with 6 vertices and 11 edges. This is impossible, since the maximum size of a bipartite graph with 6 vertices is 9 .

Case III: $\bar{H} \cong H_{1} \cup(n-2 \alpha-1) K_{1}, H_{1}$ is a bipartite graph of order $2 \alpha+1$. From (4.15), (4.16) and Theorem 2.2 (i), (iii) and (iv), we get $8 \alpha-10=\gamma_{1}(\bar{H})+\gamma_{2}(\bar{H})+\gamma_{\alpha+2}(\bar{H}) \leq 6 \alpha-2$. Thus, $\alpha=3$ or 4 . Then from (4.16), we see that $H_{1}$ is a bipartite graph with 7 vertices and 12 edges, i.e., $H_{1} \cong K_{3,4}(\alpha=3)$ or $H_{1}$ is a bipartite graph with 9 vertices and 24 edges $(\alpha=4)$. The second case is clearly impossible, where as the first case contradicts (4.17). 
Next we assume that the number of bipartite components of $\bar{H}$ is exactly $n-2 \alpha-1$. Then $\gamma_{2 \alpha+1}(\bar{H})>0$. Since $\left|V\left(H_{1}\right)\right| \geq 2 \alpha$, we have the following possibilities for $\bar{H}$.

$$
\bar{H} \cong\left\{\begin{array}{l}
H_{1} \cup(n-2 \alpha-2) K_{1}, \quad H_{1} \text { is a bipartite graph of order } 2 \alpha+2, \\
H_{1} \cup(n-2 \alpha-3) K_{1} \cup P_{3}, \quad H_{1} \text { is a bipartite graph of order } 2 \alpha, \\
H_{1} \cup(n-2 \alpha-2) K_{1} \cup K_{2}, \quad H_{1} \text { is non bipartite graph of order } 2 \alpha, \\
H_{1} \cup(n-2 \alpha-4) K_{1} \cup 2 K_{2}, \quad H_{1} \text { is a bipartite graph of order } 2 \alpha, \\
H_{1} \cup(n-2 \alpha-1) K_{1}, \quad H_{1} \text { is a non bipartite graph of order } 2 \alpha+1, \text { or } \\
H_{1} \cup(n-2 \alpha-3) K_{1} \cup K_{2}, \quad H_{1} \text { is a bipartite graph of order } 2 \alpha+1 .
\end{array}\right.
$$

Case $I: \bar{H} \cong H_{1} \cup(n-2 \alpha-2) K_{1}, H_{1}$ is a bipartite graph of order $2 \alpha+2$. From (4.14), we have $\gamma_{n-2 \alpha}(H)=n-2$. Therefore, by Theorem 2.2 (i), $\bar{H}$ has either $n-2 \alpha-1$ balanced bipartite components or $n-2 \alpha$ bipartite components. Since the number of bipartite components of $\bar{H}$ is exactly $n-2 \alpha-1$, all $n-2 \alpha-1$ bipartite components of $\bar{H}$ are balanced. Since $\bar{H} \cong H_{1} \cup(n-2 \alpha-2) K_{1}$ and an isolated vertex $K_{1}$ is not balanced, it follows that $n-2 \alpha-2=0$ and $H_{1}$ is a balanced bipartite component. Therefore, by (4.16), $2|E(\bar{H})|=4 \alpha(\alpha-1) \leq 2(\alpha+1)^{2}$. This implies that $\alpha=3$ or 4 . If $\alpha=3$, then $H_{1}$ is balanced bipartite graph with 8 vertices and 12 edges, and so $H_{1}$ is a graph obtained from $K_{4,4}$ by deleting 4 edges. In this case, by Sage [23], it can be seen that no such graphs satisfies (4.17). If $\alpha=4$, then $H_{1}$ is a balanced bipartite graph with 10 vertices and 24 edges, and so $H_{1} \cong K_{5,5} \backslash\{e\}$, where $e$ is an edge in $K_{5,5}$. By Sage [23], this is not possible by (4.17).

Case II: $\bar{H} \cong H_{1} \cup(n-2 \alpha-3) K_{1} \cup P_{3}, H_{1}$ is a bipartite graph of order $2 \alpha$. From (4.14), we have $\gamma_{n-2 \alpha}(H)=n-2$. Therefore, by Theorem 2.2 (i), $\bar{H}$ has either $n-2 \alpha-1$ balanced bipartite components or $n-2 \alpha$ bipartite components. Thus, $\bar{H}$ has $n-2 \alpha-1$ balanced bipartite components as the number of bipartite components of $\bar{H}$ is exactly $n-2 \alpha-1$. Since $P_{3}$ is not balanced, we have a contradiction.

Case III: $\bar{H} \cong H_{1} \cup(n-2 \alpha-2) K_{1} \cup K_{2}, H_{1}$ is non bipartite graph of order $2 \alpha$. Since $\left|H_{1}\right|=2 \alpha$, by Theorem 2.2 (i), we have $\gamma_{2}(\bar{H}) \leq 2 \alpha-2$. From (4.15), we get $\gamma_{2}(\bar{H}) \geq 2 \alpha-2$, and hence, $\gamma_{2}(\bar{H})=2 \alpha-2$. Since $\bar{H}=H_{1} \cup(n-2 \alpha-2) K_{1} \cup K_{2}$, we have that $\bar{H}$ has 2 as its signless Laplacian eigenvalue. Since $\alpha \geq 3$, from (4.15), we have $\gamma_{2 \alpha}(\bar{H})=2 \alpha-4 \geq 2$. From (4.15), we conclude that $\gamma_{2 \alpha}(\bar{H})=2$ or $\gamma_{2 \alpha+1}(\bar{H})=2$. First we assume that $\gamma_{2 \alpha+1}(\bar{H})=2$. Then by (4.15), (4.16) and (4.17), we get

$$
\gamma_{1}(\bar{H})+\gamma_{\alpha+2}(\bar{H})=6 \alpha-10 \text { and } \gamma_{1}^{2}(\bar{H})+\gamma_{\alpha+2}^{2}(\bar{H})=20 \alpha^{2}-48 \alpha+28 .
$$

Solving these equations, we have $\gamma_{\alpha+2}(\bar{H})=3 \alpha-5-\sqrt{\alpha^{2}+6 \alpha-11}<2 \alpha-4$, a contradiction. Next we assume that $\gamma_{2 \alpha}(\bar{H})=2 \alpha-4=2$, that is, $\alpha=3$. Then $H_{1}$ is a non bipartite graph with 6 vertices and 11 edges. Using the $Q$-spectrum of connected graphs with 6 vertices and 11 edges, by Sage [23], one can easily see that all the choices for $H_{1}$ contradicts (4.15).

Case IV: $\bar{H} \cong H_{1} \cup(n-2 \alpha-4) K_{1} \cup 2 K_{2}, H_{1}$ is a bipartite graph of order $2 \alpha$. Since $\alpha \geq 3$, from (4.15), we have $\gamma_{2 \alpha}(\bar{H})=2 \alpha-4 \geq 2$. Since $\bar{H} \cong H_{1} \cup(n-2 \alpha-4) K_{1} \cup 2 K_{2}$, then $\bar{H}$ has 2 as its signless Laplacian eigenvalue with multiplicity 2 and so $\gamma_{2 \alpha}(\bar{H})=2$, that is, $2 \alpha-4=2$, that is, $\alpha=3$. From (4.16), $H_{1}$ is a bipartite graph with 6 vertices and 10 edges. This is not possible, since there exists no bipartite graph with 6 vertices and 10 edges.

Case $V: \bar{H} \cong H_{1} \cup(n-2 \alpha-1) K_{1}, H_{1}$ is a non bipartite graph of order $2 \alpha+1$. From (4.14), we have $\gamma_{n-2 \alpha}(H)=n-2$. Therefore, by Theorem 2.2 (i), $\bar{H}$ has either $n-2 \alpha-1$ balanced bipartite components 
or $n-2 \alpha$ bipartite components. Thus, $\bar{H}$ has $n-2 \alpha-1$ balanced bipartite components as the number of bipartite components of $\bar{H}$ is exactly $n-2 \alpha-1$. Since an isolated vertex $K_{1}$ is not balanced, in this case, we must have $n-2 \alpha=1$, a contradiction as $n-2 \alpha \geq 2$.

Case VI: $\bar{H} \cong H_{1} \cup(n-2 \alpha-3) K_{1} \cup K_{2}, H_{1}$ is a bipartite graph of order $2 \alpha+1$. From (4.14), we have $\gamma_{n-2 \alpha}(H)=n-2$. Therefore, by Theorem 2.2 (i), $\bar{H}$ has either $n-2 \alpha-1$ balanced bipartite components or $n-2 \alpha$ bipartite components. Since $\bar{H}=H_{1} \cup(n-2 \alpha-3) K_{1} \cup K_{2}$ and an isolated vertex $K_{1}$ is not balanced, it follows that $n-2 \alpha=3$ and $H_{1}$ is a balanced bipartite component of order $2 \alpha+1$. This case is not possible because a balanced bipartite graph has even number of vertices.

\section{REFERENCES}

[1] R. Boulet. Disjoint unions of complete graphs characterized by their Laplacian spectrum. Electron. J. Linear Algebra, 18:773-783, 2009.

[2] A.E. Brouwer and W.H. Haemers. Spectra of Graphs. Springer, Berlin, 2012.

[3] C. Bu and J. Zhou. Signless Laplacian spectral characterization of the cones over some regular graphs. Linear Algebra Appl., 436:3634-3641, 2012.

[4] M. Cámara and W.H. Haemers. Spectral characterizations of almost complete graphs. Discrete Appl. Math., 176:19-23, 2014.

[5] D. Cvetković, P. Rowlinson, and S. Simić. An Introduction to the Theory of Graph Spectra. Cambridge University Press, Cambridge, 2010.

[6] D. Cvetković, P. Rowlinson, and S. K. Simić. Signless Laplacians of finite graphs. Linear Algebra Appl., 423:155-171, 2007.

[7] K.C. Das and M. Liu. Complete split graph determined by its (signless) Laplacian spectrum. Discrete Appl. Math., 205:45-51, 2016.

[8] K.C. Das and M. Liu. Kite graphs determined by their spectra. Appl. Math. Comput., 297:74-78, 2017.

[9] K.C. Das and S.A. Mojallal. Relation between signless Laplacian energy, energy of graph and its line graph. Linear Algebra Appl., 483:91-107, 2016.

[10] M.A.A. de Freitas, N.M.M. de Abreu, R.R. Del-Vecchio, and S. Jurkiewicz. Infinite families of Q-integral graphs. Linear Algebra Appl., 432:2352-2360, 2010.

[11] L.S. de Lima and V. Nikiforov. On the second largest eigenvalue of the signless Laplacian. Linear Algebra Appl., 438:1215$1222,2013$.

[12] H. Günthard and H. Primas. Zusammenhang von Graphentheorie und MO-Theorie von Molekeln mit Systemen konjugierter Bindungen. Helv. Chim. Acta, 39:1645-1653, 1956.

[13] I. Gutman, M. Robbiano, E.A. Martins, D.M. Cardoso, L. Medina, and O. Rojo. Energy of line graphs. Linear Algebra Appl., 433:1312-13231, 2010.

[14] C. He and E.R. van Dam. Laplacian spectral characterization of roses. Linear Algebra Appl., 536:19-30, 2018.

[15] R.A. Horn and C.R. Johnson. Matrix Analysis. Cambridge University Press, New York, 2012.

[16] S. Huang, J. Zhou, and C. Bu. Signless Laplacian spectral characterization of graphs with isolated vertices. Filomat, 30:3689-3696, 2016.

[17] Y.-L. Jin and X.-D. Zhang. Complete multipartite graphs are determined by their distance spectra. Linear Algebra Appl., 448:285-291, 2014.

[18] H.Q. Lin, Y. Hong, J.F. Wang, and J.L. Shu. On the distance spectrum of graphs. Linear Algebra Appl., 439:1662-1669, 2013.

[19] M. Liu. Some graphs determined by their (signless) Laplacian spectra. Czechoslovak Math. J., 62:1117-1134, 2012.

[20] M. Liu, B. Liu, and F. Wei. Graphs determined by their (signless) Laplacian spectra. Electron. J. Linear Algebra, $22: 112-124,2011$.

[21] X. Liu and P. Lu. Signless Laplacian spectral characterization of some joins. Electron. J. Linear Algebra, 30:443-454, 2014.

[22] M. Liu, Y. Zhu, H. Shan, and K.C. Das. The spectral characterization of butterfly-like graphs. Linear Algebra Appl., 513:55-68, 2017.

[23] W.A. Stein et al. Sage Mathematics Software (Version 6.8). The Sage Development Team, http://www.sagemath.org, USA, 2015.

[24] H. Topcu, S. Sorgun, and W.H. Haemers. On the spectral characterization of pineapple graphs. Linear Algebra Appl., 507:267-273, 2016. 
Electronic Journal of Linear Algebra, ISSN 1081-3810

A publication of the International Linear Algebra Society

Volume 36, pp. 461-472, July 2020.

[25] E.R. van Dam and W.H. Haemers. Which graphs are determined by their spectra. Linear Algebra Appl., 373:241-272, 2003.

[26] E.R. van Dam and W.H. Haemers. Developments on spectral characterizations of graphs. Discrete Math., 309:576-586, 2009.

[27] L. Xu and C. He. On the signless Laplacian spectral determination of the join of regular graphs. Discrete Math. Algorithm. Appl., 6:1450050, 2014.

[28] J. Xue, R. Liu, and H. Jia. On the distance spectrum of trees. Filomat, 30:1559-1565, 2016.

[29] J. Zhou and C. Bu. Laplacian spectral characterization of some graphs obtained by product operation. Discrete Math., 312:1591-1595, 2012 